Proceedings

\title{
Electron Capture Detector with Non-Radioactive Electron Source ${ }^{+}$
}

\author{
Erik Bunert *, Ansgar T. Kirk, Jens Oermann and Stefan Zimmermann \\ Department of Sensors and Measurement Technology, Institute of Electrical Engineering and Measurement \\ Technology, Leibniz Universität Hannover, Appelstr. 9A, 30167 Hannover, Germany; \\ kirk@geml.uni-hannover.de (A.T.K.); jens.oermann@gmx.de (J.O.); \\ zimmermann@geml.uni-hannover.de (S.Z.) \\ * Correspondence: bunert@geml.uni-hannover.de; Tel.: +49-511-762-3761 \\ † Presented at the Eurosensors 2017 Conference, Paris, France, 3-6 September 2017.
}

Published: 16 August 2017

\begin{abstract}
Gas chromatographs equipped with electron capture detectors (ECD) are widely used for the analysis of electron affine substances. Achieving limits of detection in the low pptv-range, electron capture detectors are the most sensitive detectors available for such compounds. Based on their operating principle, they require free electrons at atmospheric pressure, which are usually generated by using a $\beta$-decay. However, the use of radioactive materials leads to regulatory restrictions regarding purchase, operation and disposal. Here, we present a new electron capture detector using a non-radioactive electron source, which is not subject to these limitations and offers further advantages such as adjustable and higher electron densities and energies.
\end{abstract}

Keywords: electron capture detector (ECD); gas chromatography; GC detector; non-radioactive electron source; non-radioactive ECD

\section{Introduction}

Electron capture detectors (ECD) have become the most important gas chromatography (GC) detectors for the detection of electron affine compounds since their advent more than 55 years ago [1]. Although many attempts have been made to replace the radioactive source used to generate thermalized electrons by ionizing the carrier gas [2], only the pulsed helium discharge has seen some degree of application. However, in other fields, for example ion mobility spectrometry, radioactive electron sources have been successfully replaced by non-radioactive electron sources. In recent years, we developed a compact, hermetically sealed non-radioactive electron source [3]. It generates free electrons through thermionic emission in a vacuum chamber. These electrons are then accelerated and can partially transmit through a $300 \mathrm{~nm}$ thin silicon nitride membrane to atmospheric pressure. By varying the acceleration voltage and filament heating current, the electron energy and density can be controlled. In first fundamental investigations we attached our non-radioactive electron source to a small ionization chamber with a faraday plate detector [4]. Here, we investigated the influence of the gas flow configuration and the operating parameters of the electron source on the sensitivity and noise level using a constant 1,1,2-trichloroethane concentration of $50 \mathrm{ppb}_{\mathrm{v}}$. In this work we present further investigations of the influence of the remaining operating parameters, such as the collector voltage or lower gas flows, on the analytical performance of our non-radioactive ECD.

\section{Experimental}

Our non-radioactive electron source, which is shown in Figure 1a, is attached to a small ionization chamber manufactured from polyether ether ketone (PEEK) as shown in Figure $1 \mathrm{~b}$. The electron source consists of a hot filament, a control grid and a getter and is sealed with a $300 \mathrm{~nm}$ thin 
silicon nitride $\left(\mathrm{Si}_{3} \mathrm{~N}_{4}\right)$ membrane. The membrane is $1.5 \mathrm{~mm} \times 1.5 \mathrm{~mm}$ and transparent for electrons with sufficient electron energy [5]. The generated free electrons are accelerated by applying an adjustable acceleration voltage $U_{\text {acc }}$ between the filament and the acceleration electrode and can partially transmit through the $\mathrm{Si}_{3} \mathrm{~N}_{4}$ membrane to atmospheric pressure. By varying the acceleration voltage and filament heating current, the electron energy and density can be controlled.

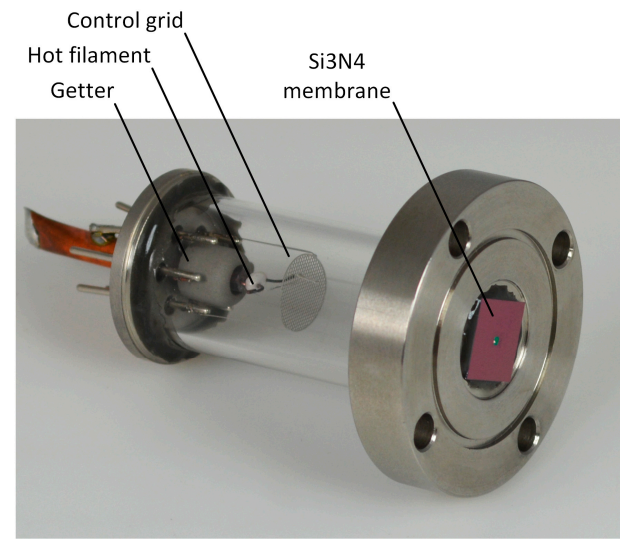

(a)

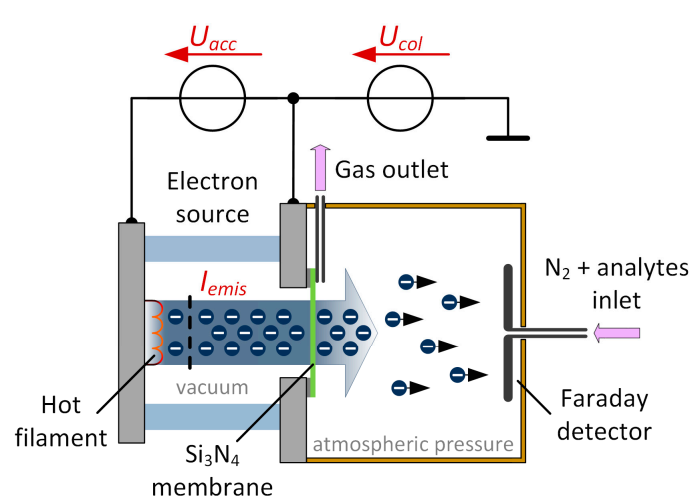

(b)

Figure 1. Photo of our hermetically sealed non-radioactive electron source based on thermionic electron emission (a) and concept of the electron capture detector with axial gas flow (b) employing the non-radioactive electron source.

Inside the ionization chamber a faraday plate detector is placed. By applying a constant collector voltage $U_{\mathrm{col}}(\mathrm{DC}$ mode) between the acceleration electrode of the electron source and the Faraday detector, the generated electrons can be collected at the Faraday detector. This constitutes a constant detector current, which is amplified by our modified transimpedance amplifier [6] with reduced gain and thus an increased input range and measured by a Keysight $34461 \mathrm{~A}$ multimeter. The carrier gas containing the analyte initially flows with a constant flow of $30 \mathrm{mls} / \mathrm{min}$ through the ionization chamber. Now, the electron-capturing compounds in the carrier gas can react with the free, thermalized electrons, leading to a decreased detector current [7].

The carrier gas containing the analyte flows from the Faraday detector towards the electron source as depicted in Figure $1 \mathrm{~b}$ and described in our previous work [4]. Furthermore, a constant acceleration voltage of $U_{\text {acc }}=10 \mathrm{kV}$ and an emission current of $I_{\text {emis }}=40 \mathrm{nA}$ is used. For generating a constant analyte vapor concentration, the analyte is filled into a permeation vial heated to $35^{\circ} \mathrm{C}$ in a permeation oven purged with a constant flow of $600 \mathrm{mls} / \mathrm{min}$ of nitrogen. An adjustable fraction of this gas is diluted with pure nitrogen for generating different analyte concentrations in the carrier gas. For the following characterization, we used 1,1,2-trichloroethane (CAS: 79-00-5) and sevoflurane (CAS: 28523-86-6) as analyte, purchased from Sigma Aldrich (St. Louis, MI, USA).

\section{Results and Discussion}

First, we investigated the influence of the collector voltage $U_{\text {col }}$ on the achievable sensitivity of our setup. Therefore, the absolute detector current is measured for different collector voltages between $U_{\mathrm{col}}=1 \mathrm{~V}$ and $U_{\mathrm{col}}=180 \mathrm{~V}$. For determining the respective sensitivities, the described collector voltage sweep is repeated, but with an 1,1,2-trichloroethane concentration of $230 \mathrm{ppb}_{\mathrm{v}}$. The corresponding results are shown in Figure 2a. The analyte reactions with the free electrons cause a decrease of the detector current. Thus, the difference between the two curves corresponds to the sensitivity of this setup, which is plotted in the inset in Figure 2a. The detector currents for both curves saturate to the same value of $I_{\text {det, sat }}=17.2 \mathrm{nA}$ for higher collector voltages, independent of the analyte concentration. In addition, the sensitivity has a maximum value of $S_{\max }=2.9 \mathrm{pA} / \mathrm{ppb}$ at a collector voltage of $U_{\text {col }}=75 \mathrm{~V}$. 


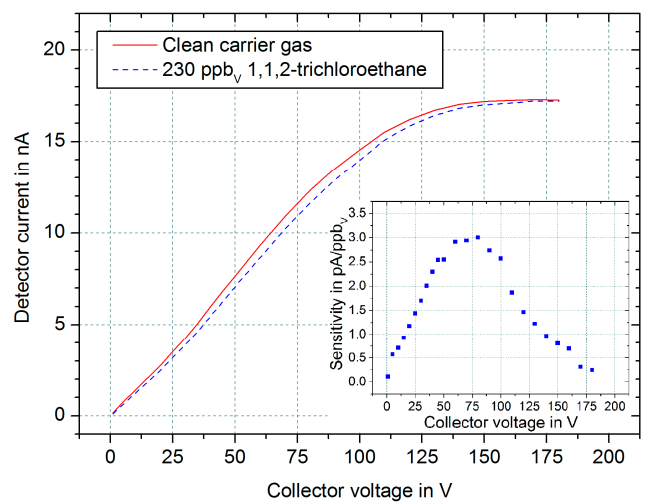

(a)

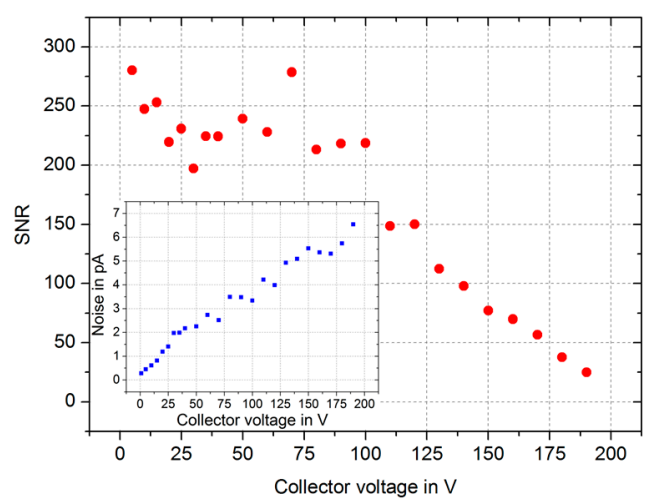

(b)

Figure 2. (a) Detector signals with pure nitrogen as carrier gas (solid red line) and nitrogen containing $230 \mathrm{ppb}_{\mathrm{v}}$ 1,1,2-trichloroethane (blue dashed line) over collector voltage; inset: resulting sensitivity (blue squares) calculated by difference and division by the gas concentration; (b) signal-to-noise ratio over collector voltage; inset: standard deviation $\sigma$ of the measured noise (blue squares).

However, not the sensitivity alone but rather a high signal-to-noise ratio (SNR) is important for a good limit of detection (LoD). Therefore, the SNR is calculated by dividing the sensitivity by the noise of the detector signal for a measuring time of $200 \mathrm{~ms}$ depending on the collector voltage. The results in Figure $2 \mathrm{~b}$ show that the noise (inset) increases linear with higher collector voltages. Thus, the SNR remains above a value of 200 up to $U_{\text {col }}=75 \mathrm{~V}$, because the sensitivity increases also in this collector voltage range. For higher collector voltages, however, the SNR decreases significantly. Furthermore, for each repetition of these measurement the SNR has two maxima of 280 at $U_{\text {col }}=5 \mathrm{~V}$ and $U_{\mathrm{col}}=75 \mathrm{~V}$. Therefore, in the following measurements a collector voltage $U_{\mathrm{col}}=5 \mathrm{~V}$ is used because of its significantly decreased instrumental effort.

Now, the influence of the carrier gas flow on the resulting signal amplitude is investigated. Therefore, we performed measurements with 1,1,2-trichloroethane and sevoflurane at various concentrations for carrier gas flows of 5,10 and $30 \mathrm{mls} / \mathrm{min}$. The results in Figure 3 show a slightly decreased sensitivity with lower carrier gas flows. A possible explanation for this can be that not all charged analyte molecules can be flushed out by the lower gas flows. Thus, not only the electrons but also the charged analyte molecules are detected, which reduce the signal amplitude. Furthermore, the typical non-linearity of the signal amplitude of electron capture detectors in DC mode $[8,9]$ with increasing concentrations is recognizable.

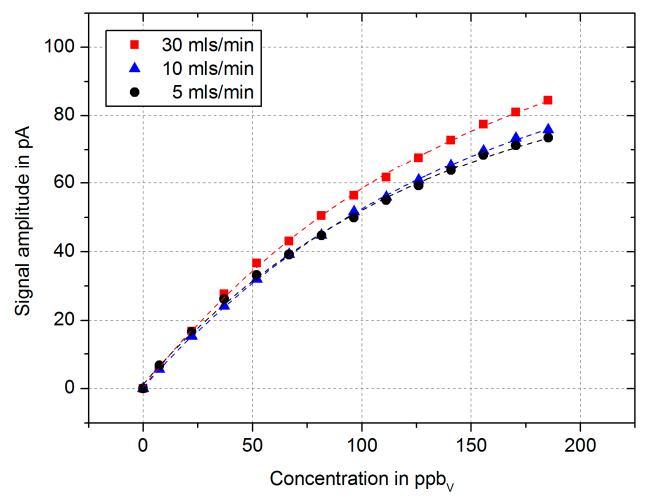

(a)

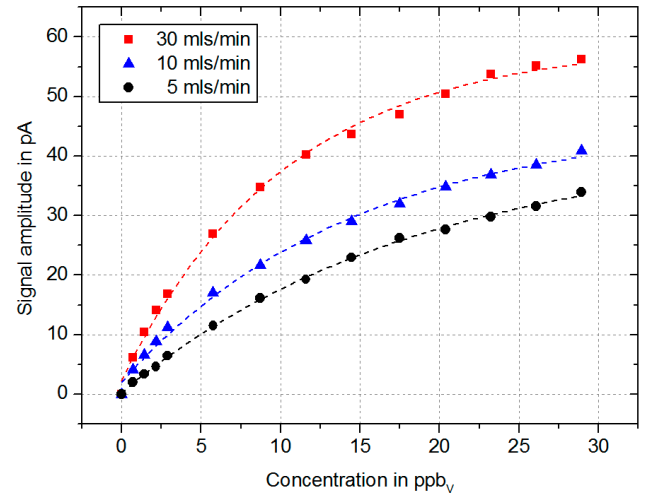

(b)

Figure 3. Resulting signal amplitudes over analyte concentration for 1,1,2-trichloroethane (a) and sevoflurane (b) for carrier gas flows of 5, 10 and $30 \mathrm{mls} / \mathrm{min}$. (a) 1,1,2-trichloroethane; (b) sevoflurane.

Based on the measured linear signal increase of these measurements at low concentrations, we determined the limit of detection (LoD) for 1,1,2-trichloroethane and sevoflurane depending on the 
carrier gas flow. The LoD is defined as three times the standard deviation $\sigma$ of the measured noise. The calculated LoDs are listed in Table 1 for a measuring time of $200 \mathrm{~ms}$. These are comparable to the typical limit of detection using an ECD with the radioactive counterpart [10].

Table 1. Limits of Detection (LoD) for 1,1,2-trichloroethane and sevoflurane for carrier gas flows between $5 \mathrm{mls} / \mathrm{min}$ and $30 \mathrm{mls} / \mathrm{min}$ for a measuring time of $200 \mathrm{~ms}$ and $U_{\text {col }}=5 \mathrm{~V}$.

\begin{tabular}{ccc}
\hline Carrier Gas Flow & LoD for 1,1,2-trichloroethane & LoD for Sevoflurane \\
\hline $5 \mathrm{mls} / \mathrm{min}$ & $1.2 \mathrm{ppb}_{\mathrm{v}}$ & $450 \mathrm{ppt}_{\mathrm{v}}$ \\
$10 \mathrm{mls} / \mathrm{min}$ & $1.1 \mathrm{ppb}_{\mathrm{v}}$ & $340 \mathrm{ppt}_{\mathrm{v}}$ \\
$30 \mathrm{mls} / \mathrm{min}$ & $1.0 \mathrm{ppb}_{\mathrm{v}}$ & $190 \mathrm{ppt}_{\mathrm{v}}$ \\
\hline
\end{tabular}

\section{Conclusions}

In this work we present further investigations of the analytical performance of an electron capture detector equipped with our non-radioactive electron source based on the collector voltage and the carrier gas flow. The results show two maximum values of the SNR at collector voltages of $U_{\text {col }}=5 \mathrm{~V}$ and $U_{\text {col }}=75 \mathrm{~V}$. Therefore, the lower voltage is preferred because of the reduced electronic requirements. Furthermore, we found that the limits of detection for 1,1,2-trichloroethane and sevoflurane is comparable to electron capture detectors with radioactive electron source, but slightly decrease with lower carrier gas flows. These limits could be improved by using a transimpedance amplifier with increased gain at the cost of a reduced input range, leading to an increased SNR.

Conflicts of Interest: The authors declare no conflict of interest.

\section{References}

1. Lovelock, J.E.; Lipsky, S.R. Electron Affinity Spectroscopy-A New Method for the Identification of Functional Groups in Chemical Compounds Separated by Gas Chromatography 1. J. Am. Chem. Soc. 1960, $82,431-433$.

2. Wentworth, W.E.; D'Sa, E.D.; Cai, H.; Stearns, S. Environmental Applications of the Pulsed-Discharge Electron-Capture Detector. J. Chromatogr. Sci. 1992, 30, 478-485.

3. Cochems, P.; Kirk, A.T.; Bunert, E.; Runge, M.; Goncalves, P.; Zimmermann, S. Fast pulsed operation of a small non-radioactive electron source with continuous emission current control. Rev. Sci. Instrum. 2015, 86, 65102.

4. Bunert, E.; Kirk, A.T.; Oermann, J.; Zimmermann, S. An Electron Capture Detector Based on a NonRadioactive Electron Source. In Proceedings of the AMA Conferences 2017 with SENSOR and IRS2: SENSOR 2017, Messezentrum, Germany, 30 May-1 June 2017; AMA Service GmbH: Wunstorf, Germany, 2017; pp. 627-631.

5. Bunert, E.; Heptner, A.; Reinecke, T.; Kirk, A.T.; Zimmermann, S. Shutterless ion mobility spectrometer with fast pulsed electron source. Rev. Sci. Instrum. 2017, 88, 24102.

6. Cochems, P.; Kirk, A.T.; Zimmermann, S. In-circuit-measurement of parasitic elements in high gain high bandwidth low noise transimpedance amplifiers. Rev. Sci. Instrum. 2014, 85, 124703.

7. Zlatkis, A.; Poole, C.F. Electron Capture: Theory and Practice in Chromatography; Elsevier Scientific Pub. Co.: Amsterdam, The Netherlands; New York, NY, USA, 1981.

8. Maggs, R.J.; Joynes, P.L.; Davies, A.J.; Lovelock, J.E. Electron capture detector. New mode of operation. Anal. Chem. 1971, 43, 1966-1971.

9. Wagner, P.D.; Naumann, P.F.; Laravuso, R.B. Simultaneous measurement of eight foreign gases in blood by gas chromatography, (eng). J. Appl. Physiol. 1974, 36, 600-605.

10. Barani, F.; Dell'Amico, N.; Griffone, L.; Santoro, M.; Tarabella, C. Determination of Volatile Organic Compounds by Headspace Trap. J. Chromatogr. Sci. 2006, 44, 625-630. 